\title{
Flow-induced energetic bounds to growth in an intertidal sea anemone
}

\author{
Bryce D. Wolcott*, Brian Gaylord \\ Marine Science Institute, University of California, Santa Barbara, California 93106, USA
}

\begin{abstract}
General models exploring patterns of maximal size in indeterminately growing, benthic marine organisms have often focused on potential scaling limits associated with either internal energetics or external physical factors such as hydrodynamic force. Rarely, however, have these broadstrokes models considered explicitly the possibility that interactions between physiological energy balances and environmental fluid motion might alter the underlying scaling relationships. Here we examine, using simple principles of allometry, the fundamental energetics of a common intertidal sea anemone Anthopleura elegantissima in the context of the rapid flows that characterize its native habitat. We find that fluid mechanical distortion of the feeding apparatus of this species modifies the scaling of predicted energy intake across size, creating a possible mismatch with its expected metabolic requirements, and potentially placing a limit on growth. Such a limit would not be expected from standard metabolic or fluid dynamic arguments applied in isolation, reiterating the often-overlooked importance of an organism's structural design and its associated mechanical response to flow.
\end{abstract}

KEY WORDS: Allometry - Anthopleura elegantissima - Energetics - Hydrodynamic forces · Flow-induced limits $\cdot$ Indeterminate growth $\cdot$ Maximal size $\cdot$ Scaling

\section{INTRODUCTION}

Individuals of many coastal marine species exhibit smaller mean adult size in locations characterized by higher levels of habitat stress. This pattern has been observed in the face of stressors as diverse as elevated temperature/salinity, desiccation, scarce resources, and exposure to extreme hydrodynamic forces (e.g. Lewis 1968, Harger 1970, Connell 1972, Paine 1976, Sebens 1980, Harris 1991). Such relationships have prompted the development of models that attempt to predict size in indeterminately growing (sensu Sebens 1987) intertidal or subtidal organisms as a function of various indices of environmental suitability. In benthic shoreline species, 2 models in particular have been commonly invoked to explain patterns of size. Each incorporates its own set of underlying assumptions.

${ }^{*}$ Present address: Department of Integrative Biology, University of California, Berkeley, California 94720-3140, USA. Email: bwolcott@socrates.berkeley.edu
One of the more widely applied general models (Sebens 1987) focuses on energetic tradeoffs. It predicts that organisms should grow to a size where the difference between the rate of energy intake and metabolic cost is maximal, which should allow for the greatest accumulation of surplus energy for reproduction over time. This assumes that there is sufficient space within the organism to enable conversion of all excess energy into reproductive tissue. In organisms where the amount of free body space constrains gonad volume, organisms should grow somewhat larger to maximize reproductive output. This basic model neglects implicitly any potential variation in mortality with size.

A second general model, in contrast, assumes that energetics play a less stringent role in limiting size than increases in mortality with size (Denny et al. 1985, Gaylord et al. 1994). In this scenario (one which has been applied predominantly to intertidal plants and animals subjected to severe flow forces), greater size enhances reproductive potential in organisms 
with constrained gonad volume as above, but is offset at sufficiently large size by a decrease in the probability of survivorship. This overall concept derives from hydrodynamic arguments that suggest surfzone plants and animals become more vulnerable to damage or dislodgment by breaking waves as these organisms get bigger. The importance of the dominant flow factor leading to such scaling mismatches, however, has been recently reexamined and discounted (Gaylord 2000).

Of course, physiological energy balances and flow effects need not operate independently, and multiple model elements may typically apply. Numerous examples exist, for instance, where fluid motion affects rates of food acquisition. Sessile suspension feeders that extract their diet from passing water provide a prime case in point (e.g. Patterson 1991, Helmuth \& Sebens 1993, Lesser et al. 1994, Hart \& Strathmann 1995, Eckman \& Okamura 1998). However, while the widespread relevance of flow is well recognized, generalized energetics-based models of optimal size often employ simplified parameterizations of the effects of water motion. Such models typically acknowledge the obvious dependence of particle flux on fluid velocity, but neglect subtleties such as the capacity for flow to alter the underlying allometric scaling of energy intake with size. While at times this assumption may be appropriate, at other times it may not, and our objective here is to examine possible failings in more detail. With this aim, we focus on a case example of a sea anemone that may experience different potential rates of energy intake across size purely due to passive flowinduced structural deformation. Our findings demonstrate that fluid motion might, under certain circumstances, indirectly produce constraints on size, even when standard metabolic scaling relationships or patterns of disturbance resistance alone would suggest otherwise.

\section{MECHANISMS OF PARTICLE CAPTURE}

An understanding of organism energetics across size requires at its most basic level an estimate of the rates at which food is acquired. In general, 3 primary mechanisms dominate prey capture by suspension-feeding animals in marine habitats characterized by strongly advective flows (Rubenstein \& Koehl 1977, Shimeta \& Jumars 1991).

Gravitational deposition. Particles that are denser than seawater fall vertically due to gravity and can be collected by horizontally oriented surfaces, at rates given by:

$$
F_{\mathrm{g}}=C w_{\mathrm{s}} A_{\mathrm{g}}
$$

where $C$ is the concentration of particles in the fluid, $W_{\mathrm{s}}$ is the particle sinking velocity, and $A_{\mathrm{g}}$ is the projected area of a particle collector facing gravity, including an $r_{\mathrm{p}}$-wide strip around the collector's periphery, where $r_{\mathrm{p}}$ is the particle radius.

Inertial impaction. Moving particles that are denser than the fluid will also deviate from curved flow trajectories as a consequence of their excess inertia. If in doing so they pass within 1 particle radius of an organism, they can be captured. In rapid surf-zone flows, particles often acquire substantial momentum such that their stopping distances exceed greatly the radius of an organism's particle collector. Under such conditions, secondary terms involving the degree of excess density of particles can be eliminated (see Shimeta \& Jumars 1991 for a full discussion), and encounter rates by inertial impaction can be approximated by:

$$
F_{\mathrm{i}}=C u A_{\mathrm{i}}
$$

where $u$ is the local flow velocity past the collector, and $A_{\mathrm{i}}$ is the projected area of the collector facing $u$. Note that (unlike in the case for gravitational deposition) $A_{\mathrm{i}}$ does not include the $r_{\mathrm{p}}$-wide strip surrounding the collector, since Eq. (2) excludes particles that would already impinge due to direct interception (outlined below), regardless of their density.

Direct interception. Even neutrally buoyant particles can be captured if they follow streamlines that pass within one particle radius of a collector. Encounter rates due to this mechanism are roughly:

$$
F_{\mathrm{d}}=\operatorname{Cur}_{\mathrm{p}} l_{\mathrm{c}}
$$

where $I_{\mathrm{c}}$ is the perimeter length of the collector in a plane perpendicular to flow. This expression, while superficially different from that given by (for example) Shimeta \& Jumars (1991), reduces to the traditional form if the usual cylindrical collector is assumed. Note that because capture efficiency of particles is rarely $100 \%$, the encounter rates for all 3 mechanisms above represent upper bounds on rates of actual food acquisition.

\section{MATERIALS AND METHODS}

Flow tank measurements. Individuals of Anthopleura elegantissima, a common intertidal sea anemone along much of the west coast of North America (Morris et al. 1980), were collected from local populations near the Marine Science Institute of the University of California in Santa Barbara. Each anemone was allowed to adhere individually to a ceramic tile and was maintained in running seawater until experimentation. Specimens ranged from 1.2 to $4.7 \mathrm{~cm}$ in dia- 
meter (tentacle tip to tentacle tip across the center of the oral disk), with a mean of $3.0 \mathrm{~cm}$. This size range is representative of individuals of this species (Hand 1955, Pearse \& Francis 2000).

A large recirculating flow tank (Vogel \& LaBarbera 1978) was constructed to examine the structural response of the anemones to fluid dynamic forces. Seawater pumped through $30 \mathrm{~cm}$ diameter PVC pipe was accelerated smoothly through a choke and transitioned into a straight, rectangular working section (Fig. 1) where freestream velocities of up to $3 \mathrm{~m} \mathrm{~s}^{-1}$ could be attained, measured by means of a pitot-static tube. These velocities approximate those found in surf-zone regions where Anthopleura elegantissima lives (Denny 1985, Gaylord 1999). Vertical profiles of velocity within the working section (Fig. 2) exhibit the classical shape of a turbulent boundary layer, as expected (Schlicting 1979).

During testing, individual anemones (attached to a ceramic tile) were placed in the flow tank and given several minutes to reach a fully expanded state in still water. The pump for the flow tank was subsequently activated, and at each of 6 predetermined freestream velocities $\left(0.0,0.3,0.6,0.9,1.2\right.$, and $\left.1.5 \mathrm{~m} \mathrm{~s}^{-1}\right)$, diameters of the anemone's tentacular crown (outer tentacle tip to outer tentacle tip parallel to the plane of the oral disk, both aligned with and normal to flow) were recorded. Grids printed on each tile allowed an observer from above to estimate (correcting for parallax) these diameters to the nearest millimeter. Observations indicated that Anthopleura elegantissima passively deforms in response to hydrodynamic forces but does not, at least over timescales of minutes, actively adjust its posture in the rapid flows present in the flow tank.

Wet mass was employed as an index of body size for use in subsequent energetic scaling analyses. Immediately following observations in the flow tank, each anemone was removed from its tile, gently squeezed dry in a clean towel, rinsed in deionized water, blotted dry, and weighed. Tsuchida \& Potts (1994) found a highly significant linear relationship $\left(\mathrm{r}^{2}=0.991, \mathrm{p}<\right.$ 0.0001) between wet and dry mass measurements using this method. Conversions based on this relation-

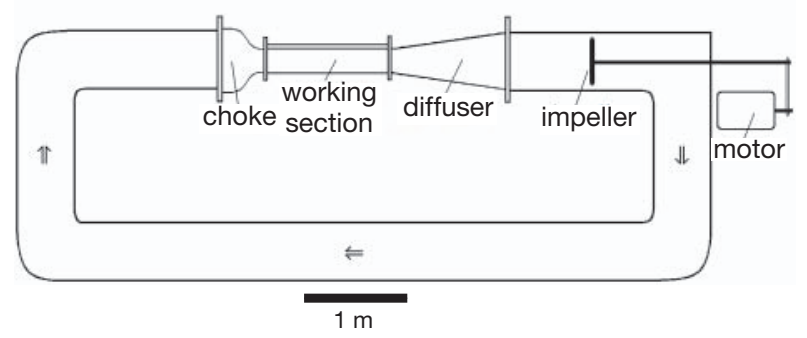

Fig. 1. Schematic of the recirculating flow tank used for the laboratory experiments

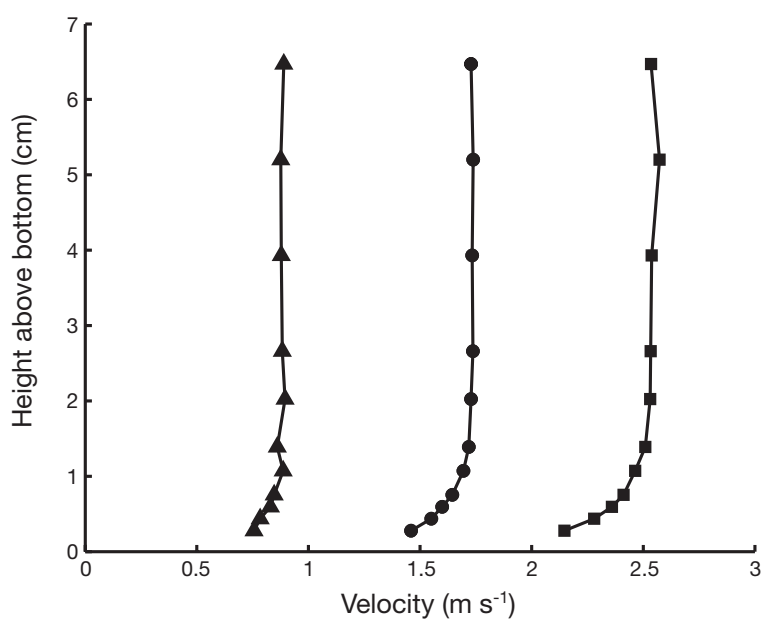

Fig. 2. Representative velocity profiles in the flow tank, for nominal (free-stream) speeds of $0.8,1.7$, and $2.5 \mathrm{~m} \mathrm{~s}^{-1}$. Note that all anemones tested in this study were taller than approximately $1 \mathrm{~cm}$, placing their deformable crowns outside the steepest part of the velocity gradient

ship therefore allow comparison with other studies of this species where size was expressed as dry mass.

Anemone feeding capacity. Potential rates of food acquisition by anemones depend on their shape and orientation relative to both flow and gravity. Two configurations in particular emerge as most relevant to this study.

The first configuration comprises an individual growing from an arbitrarily inclined substratum, with flows impinging on its oral disk from an oblique angle (Fig. 3A). This represents the most general flow/organism geometry, with components of the projected area of an anemone's oral disk and tentacles facing both the gravity vector as well as into flow. This allows all 3 major particle capture mechanisms to play

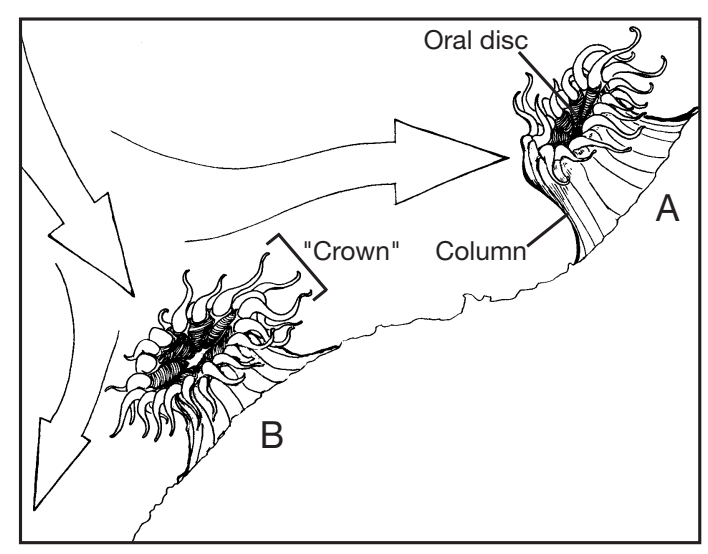

Fig. 3. The 2 major flow/anemone configurations of this study (see text) 
a role. A gross overestimate of capture potential is attained therefore by calculating maximal possible values for each mechanism. Thus, the full projected area of the oral disk plus surrounding tentacles, computed as for an ellipse, is used to compute the projected areas for $A_{\mathrm{g}}$ and $A_{\mathrm{i}}$ :

$$
\begin{gathered}
A_{\mathrm{g}}=\pi\left(r_{1}+r_{\mathrm{p}}\right)\left(r_{2}+r_{\mathrm{p}}\right) \\
A_{\mathrm{i}}=\pi r_{1} r_{2}
\end{gathered}
$$

where $r_{1}$ and $r_{2}$ are the major and minor radii of the tentacular crown, parallel to the plane of the oral disk. This calculation ignores gaps between tentacles and therefore functions as a further overestimate of projected area for smaller particles that might pass through these gaps. The linear capture dimension for direct interception, $l_{C}$ is set equal to the circumference of the tentacular crown. Because this quantity can be expressed in exact form only through the use of rather complicated elliptic integrals, we use an approximate expression provided by Ramanujan (1913):

$$
l_{\mathrm{C}}=\pi\left\{3\left(r_{1}+r_{2}\right)-\sqrt{\left(r_{1}+3 r_{2}\right)\left(3 r_{1}+r_{2}\right)}\right\}
$$

Naturally, for some flow/anemone orientations, 1 or more of the 3 capture dimensions, $A_{\mathrm{g}}, A_{\mathrm{i}}$, or $l_{\mathrm{c}}$ may become less relevant. For example, an anemone growing outwards from a vertical rock face would capture few particles via gravitational deposition, making the magnitude of $A_{\mathrm{g}}$ moot. In other situations (e.g. when fluid moves exactly parallel to the plane of the oral disk, which is the situation in the flow tank, and a subset of the configuration of Fig. 3A), inertial impaction might allow for particle delivery to outer portions of the upstream side of the tentacular crown but not to the disk proper. This could greatly reduce the actual, active magnitude of $A_{i}$. This point also reemphasizes that the use of maximal capture dimensions for all 3 mechanisms provides a highly conservative overestimate of the capacity for anemones to acquire food. A key assumption here (for this first configuration of Fig. 3A) is that there is indeed a nontrivial component of flow parallel to the plane of the oral disk. This component can apply a lateral force to the organism, an issue that will become important below where we consider the scaling consequences of flow-induced deformation.

In contrast to the situation above, the second major configuration of interest assumes that there is essentially no component of water motion parallel to the oral disk (i.e. fluid moves only in direct alignment with, and toward, the organism's oral-aboral axis; Fig. 3B). Although all 3 capture mechanisms can play a role as before, this second scenario is clearly a rather unlikely special case. It is also one that precludes the imposition of substantial lateral forces on an anemone. The reason that we consider this more restrictive configuration

Table 1. Allometric exponent, $b$, for rates of energy intake. $\mathrm{n}$ is the number of individuals measured at each velocity, SEE is the standard error of $b$, and $r$ is Pearson's correlation coefficient for the regression. Significant $p$-values ( $p<0.05$, indicated in bold) are associated with exponents that are less than 0.77 , the exponent for energetic cost. Note that $b$ is not a function of particle size in inertial impaction or direct interception

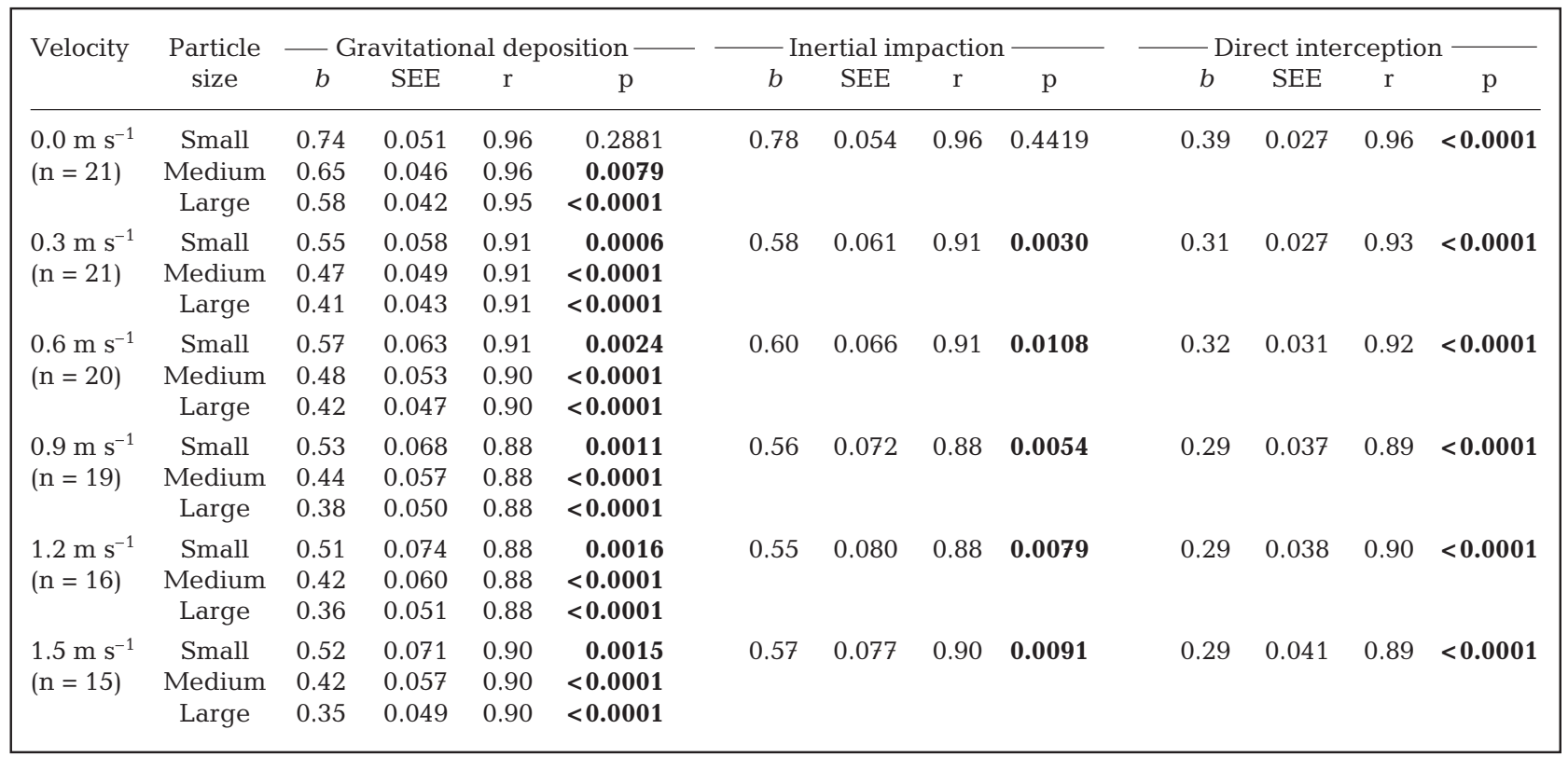


here is that it may lead to a somewhat different scaling relationship than that which arises for the more general scenario.

The capture dimensions $A_{\mathrm{g}}, A_{\mathrm{i}}$ and $l_{\mathrm{c}}$ are the only terms in the encounter rate equations (Eqs. 1 to 3 ) that vary explicitly with organism size and have the potential to affect the allometry of anemone food intake. To explore details of these size-dependent quantities and their relationship to fluid velocity, the tentacular crown diameter measurements conducted in the flow tank were divided by 2 and inserted for $r_{1}$ and $r_{2}$ in Eqs. (4), (5) \& (6). Effects of particle size $\left(r_{\mathrm{p}}\right)$ were also examined, using as a basis data from Sebens (1981), who found that Anthopleura elegantissima individuals in the field captured particles with a mean radius of $2.25 \mathrm{~mm}$, regardless of their size. Particles of this mean radius, as well as particles smaller and larger by 1 standard deviation ( $\mathrm{SD}=1.70 \mathrm{~mm}$, from Sebens' statistics), were therefore employed in Eq. (4).

For a given type of food particle, energy intake varies in direct proportion to the number of captured particles. As a consequence, the rate of energy intake, $E_{\text {intake, }}$ by each capture mechanism scales as:

$$
E_{\text {intake }} \propto\left(F_{\mathrm{g}}, F_{\mathrm{i}}, \text { or } F_{\mathrm{d}}\right) \propto\left(A_{\mathrm{g}}, A_{\mathrm{i}} \text {, or } r_{\mathrm{p}} l_{\mathrm{c}}\right) \propto a M^{b}
$$

where $M$ is organism mass, and $a$ and $b$ are constants that depend (for a given particle concentration) on the particle capture mechanism, the flow/anemone configuration, and fluid velocity. Values of $A_{\mathrm{g}}, A_{\mathrm{i}}$, and $r_{\mathrm{p}} l_{\mathrm{c}}$ were used therefore as quantitative proxies for relative rates of food intake across organism and particle size, for each of the 3 capture mechanisms. This step was accomplished by taking the natural logarithm of $A_{\mathrm{g}}, A_{\mathrm{i}}$, or $r_{\mathrm{p}} l_{\mathrm{c}}$ and performing a linear regression against the natural logarithm of anemone mass to determine $a$ and $b$ (Eq. 7) in each case. In considering the first, more common configuration of Fig. 3A, tentacular crown diameter measurements from each of the 6 flow speeds were used. This allowed for an examination of the consequences of flow-induced, lateral deformation of the oral disk and tentacles across velocities. In considering the second, more restrictive configuration, where flow parallel to the oral disk is absent (Fig. 3B), only tentacular crown diameter data from the $0.0 \mathrm{~m} \mathrm{~s}^{-1}$ flow tank runs were used, appropriate for the situation where finite lateral deformations do not arise. Due to the greater relative accuracy of the independent variable (mass), an ordinary least squares analysis (StatView 5.0, SAS Institute) was used for all of these allometric regressions (Rayner 1985). The resultant scaling of energy intake across size was then compared directly to the analogous scaling of Anthopleura elegantissima's energetic costs, $E_{\text {cost, }}$ details of which are available in the literature.

\section{RESULTS AND DISCUSSION}

\section{Anthopleura elegantissima energetics}

Under conditions of still water in the flow tank, there are no hydrodynamic forces imposed, and the $0.0 \mathrm{~m} \mathrm{~s}^{-1}$ velocity case provides a proxy for the baseline energetic scaling patterns that would arise if the organisms were not deformed in flow. With no deformation, diameters of the tentacle crown scale with mass in such a way that rates of energy intake for medium-sized (i.e. $r_{\mathrm{p}}=2.25 \mathrm{~mm}$ ) particles captured via gravitational deposition would be predicted to increase with a mass exponent $b$ of 0.65 (Table 1, Fig. 4). The analogous exponent for inertial impaction with no deformation would be 0.78 (Fig. 5), and the no-deformation exponent for direct interception would be 0.39 (Fig. 6). Only

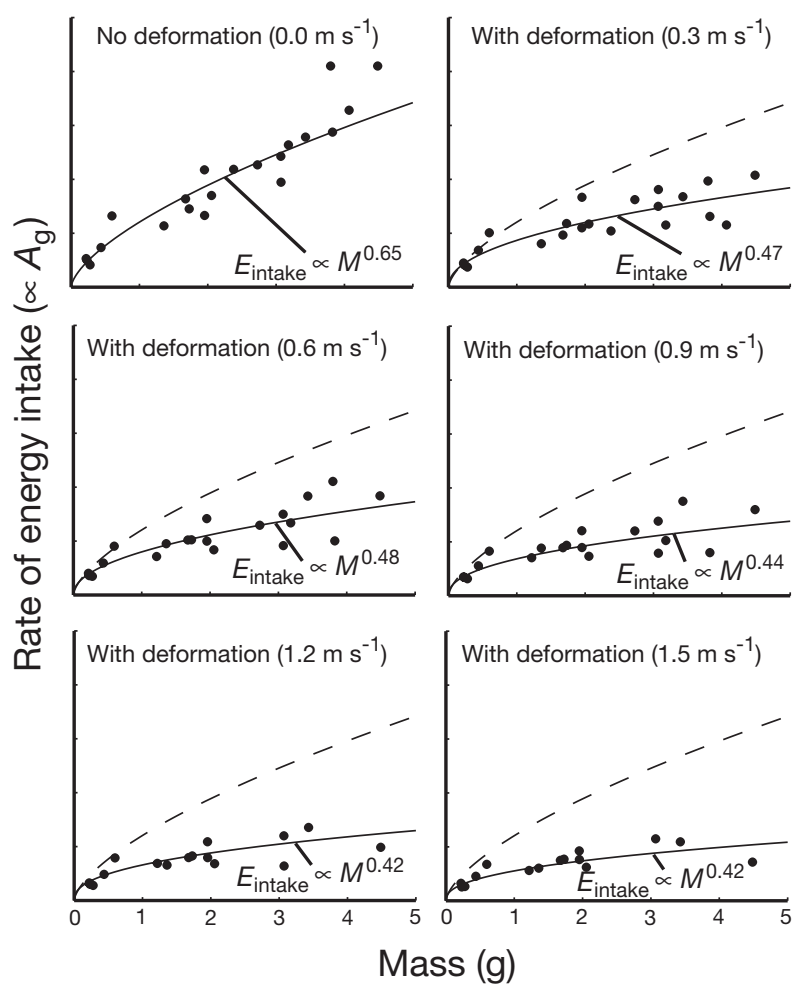

Fig. 4. Relationship between anemone mass and rates of energy intake (of medium-sized particles; $r_{\mathrm{p}}=2.25 \mathrm{~mm}$ ) by gravitational deposition, based on anemone morphologies measured under several free-stream flow rates. Note the depression of the rates of energy intake relative to the relationship that arises in the absence of flow-induced deformation (dashed line, corresponding to baseline levels estimated by means of the $0.0 \mathrm{~m} \mathrm{~s}^{-1}$ proxy case). Numerical values have been discarded from the ordinate since only the scaling with size is relevant, not absolute levels. The decrease in the number of data points at higher velocities is a result of flowinduced dislodgment of some individuals during the experiment 


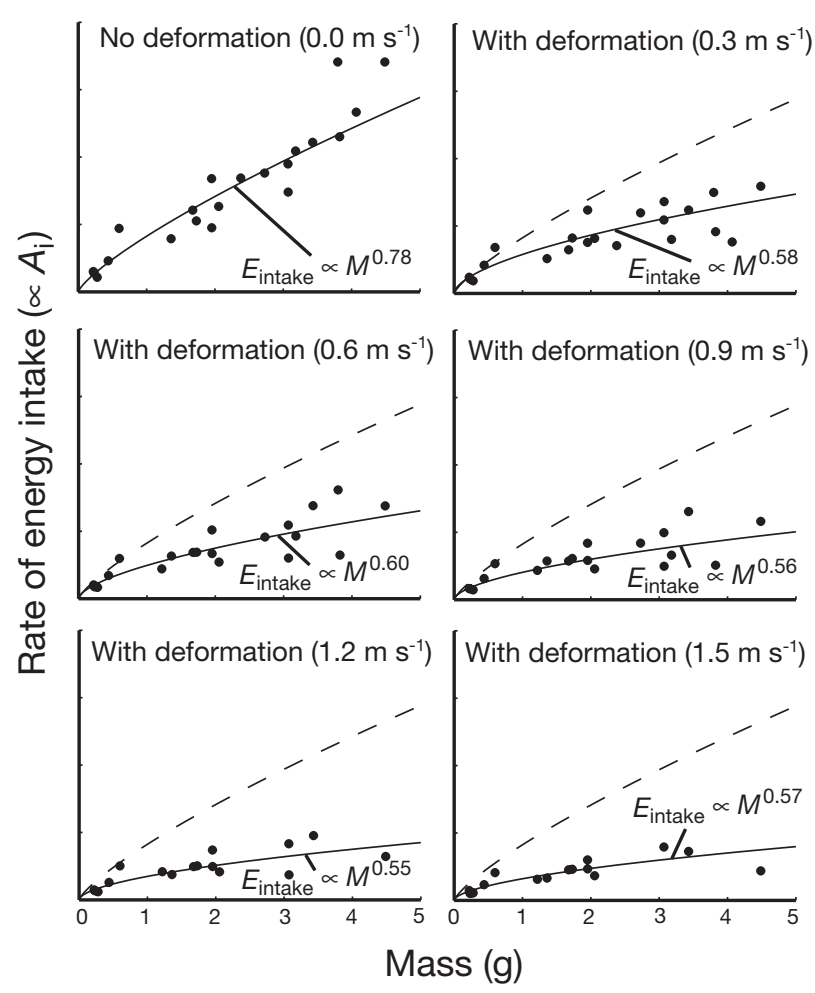

Fig. 5. Relationship between anemone mass and rates of energy intake by inertial impaction, based on anemone morphologies measured under several free-stream flow rates. Note the depression of the rates of energy intake relative to the relationship that arises in the absence of flow-induced deformation (dashed line, corresponding to baseline levels estimated by means of the $0.0 \mathrm{~m} \mathrm{~s}^{-1}$ proxy case). Numerical values have been discarded from the ordinate since only the scaling with size is relevant, not absolute levels. The decrease in the number of data points at higher velocities is a result of flow-induced dislodgment of some individuals during the experiment

2 of these values are significantly less than the mass exponent of energetic cost (0.77) quantified for Anthopleura elegantissima by Sebens (1981, see also Shick et al. 1979, Zamer 1986). Furthermore, values of $b$ for gravitational deposition also increase with decreasing particle size (Table 1). The net result is that, in the absence of flow-induced deformation, the mass exponents for intake rates of small particles $\left(r_{\mathrm{p}}=0.55 \mathrm{~mm}\right)$ by gravitational deposition, and any sized particle by inertial impaction, are not significantly less than the exponent of energetic cost. This implies that, without distortion of the tentacle crown in flow, a strict energetic limit to size should not exist in this species (Fig. 7A provides a graphical depiction of this scenario). Such a minimal-deformation scenario might be expected in exceptionally benign habitats, or as a consequence of water always moving along an anemone's oral-aboral axis only (i.e. in the second flow/anemone configuration; Fig. 3B). However, given the severe and highly variable water motion characteristic of the surf zone (Denny 1985, Gaylord 1999), at least the second of these possibilities appears unlikely.

Indeed, Anthopleura elegantissima individuals in the field are probably subjected routinely to lateral flows that deform their oral disks and tentacles. This derives from the intrinsic tendency of fluid to turn and move parallel to a solid substratum when impinging on it. Such fluid motions and their deformation effects have important implications for energetic scaling. As is shown in Figs. 4, 5 \& 6, even modest velocities can reduce capture areas and depress the mass exponents of energy intake in A. elegantissima. For all particle sizes tested, and all 3 capture mechanisms, these depressed mass exponents are significantly less than the exponent of energetic cost $(0.77)$ of this species (Table 1). This is also necessarily true of the $b$ exponent

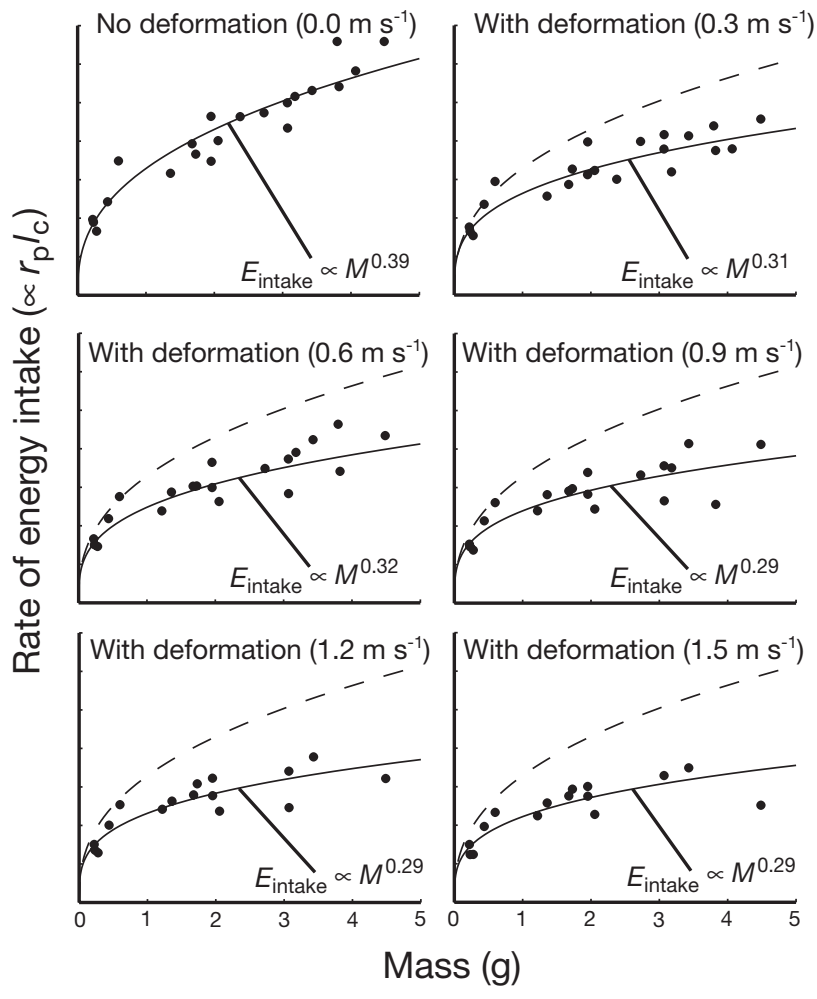

Fig. 6. Relationship between anemone mass and rates of energy intake by direct interception, based on anemone morphologies measured under several free-stream flow rates. Note the depression of the rates of energy intake relative to the relationship that arises in the absence of flow-induced deformation (dashed line, corresponding to baseline levels estimated by means of the $0.0 \mathrm{~m} \mathrm{~s}^{-1}$ proxy case). Numerical values have been discarded from the ordinate since only the scaling with size is relevant, not absolute levels. The decrease in the number of data points at higher velocities is a result of flow-induced dislodgment of some individuals during the experiment 


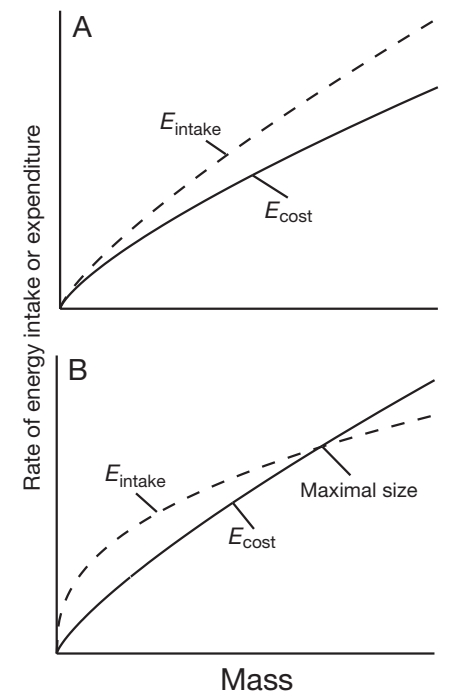

Fig. 7. Conceptual energetic scaling patterns. (A) Conditions where the mass exponent of metabolic cost does not exceed that of energetic intake (e.g. gravitational deposition of small particles or inertial impaction of any sized particles if there were no flow-induced deformation). (B) Conditions where the mass exponent of energetic intake is significantly less than that of metabolic cost (e.g. all feeding in flows of $0.3 \mathrm{~m} \mathrm{~s}^{-1}$ and faster). The size where the lines intersect in this panel sets the maximal size of an organism

associated with the summed intake from all 3 mechanisms, since for purely mathematical reasons it must fall intermediate to the overall range of values. As a consequence, in the presence of nonzero but quite common flows (flows with at least a component $>0.3 \mathrm{~m}$ $\mathrm{s}^{-1}$ parallel to an anemone's oral disk), there exists some finite size above which energetic requirements exceed rates of energy intake (i.e. $E_{\text {cost }}>E_{\text {intake) }}$. This places a potential bound on growth (Fig. 7B provides a graphical depiction) in individuals subjected to all but rather restrictive flow/anemone configurations (even flows that align exactly with an anemones oral/aboral axis may bend an organism's tentacles towards the substratum and thereby reduce capture areas). Although clonal aggregations of A. elegantissima (Hand 1955, Pearse \& Francis 2000) may reduce flow speeds to a certain extent (and thus forces imposed on individual polyps) within densely packed arrays (e.g. McFadden 1986, Anthony 1997), this feature appears unlikely to eliminate entirely the deformation response and its scaling consequences.

\section{Additional scaling considerations}

Thus far this study has considered only mechanisms of particle capture in evaluating the scaling of energy intake across size. However, many Anthopleura elegantissima individuals gain nutritive input from photosyn- thetic algal symbionts that live within their gastrodermal tissues (Muscatine 1961, Muller-Parker \& Davy 2001). Nonetheless, while such auxiliary sources of carbon are unarguably important, the dominant flux of solar energy available for photosynthesis (and thus for use by an anemone after conversion to the appropriate currency) likely scales with a projected area in much the same way as particle capture. Reduced-intensity scattered light during submergence, while potentially impinging from multiple directions, most likely represents a minor energetic contribution relative to projected area-dependent, direct insolation at low tide. This species relies heavily on prey capture regardless of symbiont activity (Shick \& Dykens 1984, Zamer \& Shick 1987).

The expressions for particle capture used in the analysis above are also simplified representations. As organisms grow and become larger (i.e. as Reynolds numbers increase; see Shimeta \& Jumars 1991), patterns of fluid motion (including velocities local to a collector surface) will change even for an individual of identical shape in the same bulk flow. However, although such changes have the potential to alter to some degree the underlying allometric relationships of energy intake, they appear unlikely to modify our major conclusions. Faster flows can also result in lateral compression of fluid trajectories as they pass the sides of a collector, increasing (in particular) encounter rates due to direct interception. Shimeta \& Jumars (1991) provide corrections for this effect (as well as for encounter due to attached downstream vortices) in adjusted 'intermediate Reynolds numbers' rate equations. These adjusted equations, however, simply approach the same general form as Eq. (2), and so are already implicitly accounted for in the above scaling calculations. This is also true for analyses that employ more intricate estimates of perimeter length $\left(l_{\mathrm{c}}\right.$ in Eq. 3) that arise from following the more convoluted path around each and every tentacle tip; such estimates are again bounded by the equation for inertial impaction.

\section{CONCLUSIONS}

Results of this study suggest that flow may affect not only the absolute rate of food acquisition in Anthopleura elegantissima, but also the underlying relationship between energy intake and anemone size. Although non-gravitational prey encounter rates increase as usual with fluid velocity (reductions in $A_{\mathrm{i}}$ and $l_{\mathrm{c}}$ do not completely counteract the absolute effects of a faster $u$ in Eqs. 2 \& 3), the dramatic (and associated) deformation-linked shifts in the relative scaling of energy intake diverge from elements of many traditional, simplified models that explore interactions between 

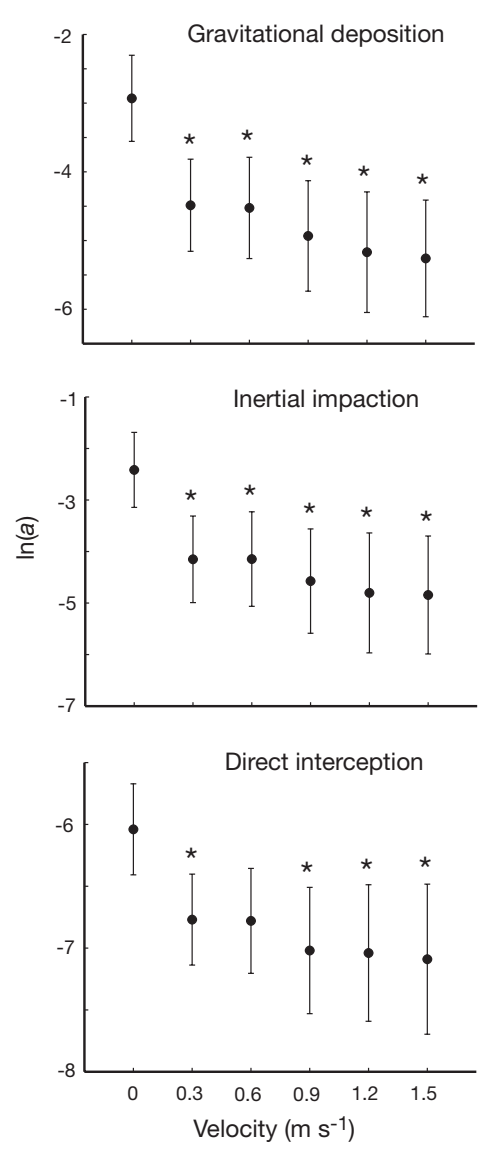

Fig. 8. The natural logarithm of the allometric coefficient, $a$, in the relationship, $E_{\text {intake }} \propto a M^{b}$, for gravitational deposition, inertial impaction, and direct interception. Error bars indicate $95 \%$ confidence intervals. Negative values reflect the fact that $a$ is typically less than 1 . Asterisks indicate velocities at which values of $a$ are significantly different $(p<0.05)$ from that at $0.0 \mathrm{~m} \mathrm{~s}^{-1}$ where there is no flow-induced anemone deformation

habitat characteristics and organism energetics. Typically, general scaling models assume that environmental factors modulate predominantly only the leading coefficient $a$ in allometric energy intake relationships (e.g. Sebens 1979, Melton 1993). Indeed, even in this study, a decreases at higher flow rates (Fig. 8). However, in contrast to traditional assumptions, the mass exponent for energy intake $b$ in $A$. elegantissima also varied with fluid velocity (Figs. 4, 5, \& 6). This less intuitive result demonstrates that environmental factors may govern not only absolute levels of food availability or prey delivery, but also the underlying, basic scaling pattern of energy intake itself. In this way, by shifting relative magnitudes of $E_{\text {intake }}$ and $E_{\text {cost }}$ across size, flow may ultimately affect the selective pressures interacting with, and operating on, body size in nature.

It may be that potential flow-mediated, indirect energetic bounds to size are present in other species as well. Hydrodynamic forces applied by crashing surf can be severe (e.g. Koehl 1977, 1984, Denny et al. 1985, Gaylord et al. 1994, 2001, Gaylord 2000), and have the potential to modulate rates of energy intake of organisms on multiple trophic levels. For example, rapid water motion appears to decrease grazing ability in herbivores (Brown \& Quinn 1988, Judge 1988), as well as foraging time in scavengers and predators (Richardson \& Brown 1990, Hahn 1998). If such inhibitory effects demonstrate size-dependent scaling, extreme flows may have the capacity to induce energetic constraints on size in an unexpectedly wide variety of organisms.

We emphasize, however, that the patterns described here are only suggestive, and are based largely on theoretical relationships. Real-world intertidal flows are complex, typically change in direction and magnitude over timescales of seconds or less, exhibit complicated spatial structure that can have non-intuitive consequences (Gaylord 2000), and are affected strongly by the microhabitats in which organisms live. Laboratory and field experiments also have demonstrated that capture rates can vary in unexpected ways due to shifts in capture location along an organism's body in different flow situations (e.g. Patterson 1984, 1991, Sebens et al. 1998). Thus, despite the suggestive nature of this study's findings, further research must examine the implications of such factors before a full reckoning of possible flow-linked bounds to size can be achieved.

Acknowledgements. J. J. Childress provided helpful advice regarding metabolic scaling, and S. D. Gaines supplied laboratory space, funds for apparatus construction, and feedback. A. Wyndham kindly and expertly created Fig. 3. Three anonymous reviewers provided helpful and extensive feedback on the manuscript. This is contribution number 88 of the Partnership for Interdisciplinary Studies of Coastal Oceans (PISCO): A Long-Term Ecological Consortium funded by the David and Lucile Packard Foundation.

\section{LITERATURE CITED}

Anthony KRN (1997) Prey capture by the sea anemone Metridium senile (L.): effects of body size, flow regime, and upstream neighbors. Biol Bull (Woods Hole) 192:73-86

Brown KM, Quinn JF (1988) The effect of wave action on growth in three species of intertidal gastropods. Oecologia 75:420-425

Connell JH (1972) Community interactions on marine rocky intertidal shores. Annu Rev Ecol Syst 3:169-192

Denny MW (1985) Wave forces on intertidal organisms: a case study. Limnol Oceanogr 30:1171-1187

Denny MW, Daniel TL, Koehl MAR (1985) Mechanical limits to size in wave swept organisms. Ecol Monogr 55:62-102

Eckman JE, Okamura B (1998) A model of particle capture by bryozoans in turbulent flow: significance of colony form. Am Nat 152:861-880 
Gaylord B (1999) Detailing agents of physical disturbance: wave-induced velocities and accelerations on a rocky shore. J Exp Mar Biol Ecol 239:85-124

Gaylord B (2000) Biological implications of surf-zone flow complexity. Limnol Oceanogr 45:174-188

Gaylord B, Blanchette CA, Denny MW (1994) Mechanical consequences of size in wave-swept algae. Ecol Monogr 64:287-313

Gaylord B, Hale BB, Denny MW (2001) Consequences of transient fluid forces for compliant benthic organisms. J Exp Biol 204:1347-1360

Hahn DR (1998) Hermit crab shell use patterns: response to previous shell experience and to water flow. J Exp Mar Biol Ecol 228:35-51

Hand C (1955) The sea anemones of Central California. Part II. The Endomyarian and Mesomyarian anemones. Wasman J Biol 13(1):37-99

Harger JRE (1970) The effect of wave impact on some aspects of the biology of sea mussels. Veliger 12(4):401-414

Harris LG (1991) Comparative ecology of subtidal actinarians from the coasts of California and the Gulf of Maine USA. Hydrobiologia 216-217:271-278

Hart MW, Strathmann RR (1995) Mechanisms and rates of suspension feeding. In: McEdward LR (ed) Ecology of marine invertebrate larvae. CRC Press, Boca Raton, FL

Helmuth B, Sebens K (1993) The influence of colony morphology and orientation to flow on particle capture by the scleractinian coral Agaricia agaricites (Linnaeus). J Exp Mar Biol Ecol 165:251-278

Judge ML (1988) The effects of increased drag on Lottia gigantea (Sowerby 1834) foraging behavior. Funct Ecol 2: 363-369

Koehl MAR (1977) Effects of sea anemones on the flow forces they encounter. J Exp Biol 69:87-105

Koehl MAR (1984) How do benthic organisms withstand moving water? Am Zool 24:57-70

Lesser MP, Witman JD, Sebens KP (1994) Effects of flow and seston availability on scope for growth of benthic suspension-feeding invertebrates from the Gulf of Maine. Biol Bull (Woods Hole) 187:319-335

Lewis JR (1968) Water movements and their role in rocky shore ecology. Sarsia 34:13-36

McFadden CS (1986) Colony fission increases particle capture rates of a soft coral: advantages of being a small colony. J Exp Mar Biol Ecol 103(1-3):1-20

Melton RH (1993) Body size evolution and the concept of alloenergetic change. J Theor Biol 164(3):321-339

Morris RH, Abbott DP, Haderlie EC (1980) Intertidal invertebrates of California. Stanford University Press, Stanford, CA

Muller-Parker G, Davy SK (2001) Temperate and tropical algal-sea anemone symbioses. Invertebr Biol 120:104-123

Muscatine L (1961) Symbiosis in marine and fresh water coelenterates. In: Lenhoff HM, Loomis WF (eds) The biology of hydra and of some other coelenterates. University of Miami Press, Coral Gables, FL, p 255-268

Paine RT (1976) Biological observations on a subtidal Mytilus californianus bed. Veliger 19(2):125-130

Patterson MR (1984) Patterns of whole colony prey capture in

Editorial responsibility: Otto Kinne (Editor),

Oldendorf/Luhe, Germany the octocoral, Alcyonium siderium. Biol Bull (Woods Hole) 167:613-269

Patterson MR (1991) The effects of flow on polyp-level prey capture in an octocoral, Alcyonium siderium. Biol Bull (Woods Hole) 180:93-102

Pearse V, Francis L (2000) Anthopleura sola, a new species, solitary sibling species to the aggregating sea anemone, A. elegantissima (Cnidaria: Anthozoa: Actiniaria: Actiniidae). Proc Biol Soc Wash 113(3):596-608

Ramanujan S (1913) Modular equations and approximations to pi. Q J Pure Appl Math 45:350-372

Rayner JMV (1985) Linear relations in biomechanics: the statistics of scaling functions. J Zool 206:415-439

Richardson TD, Brown KM (1990) Wave exposure and prey size selection in an intertidal predator. J Exp Mar Biol Ecol 142:105-120

Rubenstein DI, Koehl MAR (1977) The mechanisms of filter feeding: some theoretical considerations. Am Nat 111:981-994

Schlicting H (1979) Boundary-layer theory. McGraw-Hill, New York

Sebens KP (1979) The energetics of asexual reproduction and colony formation in bentic marine invertebrates. Am Zool 19(3):683-697

Sebens KP (1980) The regulation of asexual reproduction and indeterminate body size in the sea anemone Anthopleura elegantissima (Brandt). Biol Bull (Woods Hole) 158: 370-382

Sebens KP (1981) The allometry of feeding, energetics, and body size in three sea anemone species. Biol Bull (Woods Hole) 161:152-171

Sebens KP (1987) The ecology of indeterminate growth in animals. Annu Rev Ecol Syst 18:371-407

Sebens KP, Grace SP, Helmuth B, Maney EJ, Miles JS (1998) Water flow and prey capture by three scleractinian corals, Madracis mirabilis, Montastrea cavernosa, and Porites porites, in a field enclosure. Mar Biol 131:347-360

Shick JM, Dykens JA (1984) Photobiology of the symbiotic sea anemone Anthopleura elegantissima: photosynthesis, respriation, and behavior under intertidal conditions. Biol Bull (Woods Hole) 166:608-619

Shick JM, Brown WI, Dolliver EG, Kayar SR (1979) Oxygen uptake in sea anemones: effects of expansion, contraction, and exposure to are and the limitations of diffusion. Physiol Zool 52:50-61

Shimeta J, Jumars PA (1991) Physical mechanisms and rates of particle capture by suspension feeders. Oceanogr Mar Biol Annu Rev 29:191-257

Tsuchida CB, Potts DC (1994) The effects of illumination, food and symbionts on growth of the sea anemone Anthopleura elegantissima (Brandt, 1835). I. Ramlet growth. J Exp Mar Biol Ecol 183:227-242

Vogel S, LaBarbera M (1978) Simple flow tanks for research and teaching. BioScience 28:638-643

Zamer WE (1986) Physiological energetics of the intertidal sea anemone Anthopleura elegantissima I. Prey capture, absorption efficiency and growth. Mar Biol 92:299-314

Zamer WE, Schick JM (1987) Physiological energetics of the intertidal sea anemone Anthopleura elegantissima. II. Energy balance. Mar Biol 93(4):481-492

Submitted: March 18, 2002; Accepted: August 21, 2002

Proofs received from author(s): December 2, 2002 